Bryn Mawr College

Scholarship, Research, and Creative Work at Bryn Mawr

College

1998

\title{
Theoretical Treatment of Quasibound Resonances in Two-Color Resonant Four-Wave Mixing Spectroscopy
}

F. Di Teodoro

Elizabeth McCormack

Bryn Mawr College, emccorma@brynmawr.edu

Let us know how access to this document benefits you.

Follow this and additional works at: http://repository.brynmawr.edu/physics_pubs

Part of the Physics Commons

\section{Custom Citation}

F. Di Teodoro and E.F. McCormack, Phys. Rev. A 57, 162 (1998).

This paper is posted at Scholarship, Research, and Creative Work at Bryn Mawr College. http://repository.brynmawr.edu/physics_pubs/9 


\title{
Theoretical treatment of quasibound resonances in two-color resonant four-wave mixing spectroscopy
}

\author{
F. Di Teodoro and E. F. McCormack \\ Department of Physics, Bryn Mawr College, Bryn Mawr, Pennsylvania 19010
}

(Received 30 June 1997; revised manuscript received 10 September 1997)

\begin{abstract}
A treatment of continuum states in the application of diagrammatic perturbation theory to calculate the signal produced in two-color resonant, four-wave mixing (TC-RFWM) spectroscopy is developed. The thirdorder susceptibility is significantly modified from that obtained when considering only discrete states. To illustrate the contribution of continuum states, the line profile of a quasibound resonance arising from the configuration interaction of bound and continuum states is derived. Analytic expressions for line profiles are presented for two specific experimental implementations of TC-RFWM used in gas-phase spectroscopic studies. While the TC-RFWM line profiles are found to be very distinct from the line profiles measured in linear spectroscopic techniques, the results demonstrate the important capability to characterize the TC-RFWM line profiles in terms of the same fundamental and physically significant parameters. [S1050-2947(98)01301-8]

PACS number(s): 32.70.Jz, 39.30.+w, 42.65. $-\mathrm{k}$
\end{abstract}

\section{INTRODUCTION}

Resonant four-wave mixing (RFWM) is a nonlinear spectroscopic technique that can offer distinct advantages over linear techniques for studying the structure and dynamics of atomic and molecular systems in the gas phase. Several appealing features of RFWM have made it attractive for doing state-selective spectroscopy. It is a coherent, backgroundfree technique with favorable signal-to-noise ratios and since the signal is based only on absorption and not on a particular decay mode for detection, any excited state may be probed regardless of its decay mode (ionization, dissociation, or fluorescence). High sensitivity can be obtained for a range of pressures and number densities and low detection limits in the range $10^{9}-10^{12}$ molecules $\mathrm{cm}^{-3}$ per quantum state have been observed $[1,2]$. These attributes have led to the application of RFWM to a wide variety of investigations in atomic and molecular physics and many have been described in several informative review articles [2-5].

Four-wave mixing spectroscopy relies on the interaction of three laser beams in a medium of interest to produce a nonlinear polarization via the third-order term of the electric susceptibility $\left(\chi^{(3)}\right)[6]$. The induced polarization generates a fourth, coherent light beam that is detected as the signal. A dramatic enhancement in the signal intensity occurs whenever the frequency of the incident laser beams is resonant with a transition in the medium. Thus a spectrum can be obtained by selectively tuning the frequency of the input laser beams. Two-color (TC) RFWM is a doubly resonant implementation of resonant four-wave mixing in which two optical fields have frequencies resonant with two different transitions. TC-RFWM techniques have received considerable attention due to their state-selective capabilities and recent results include studies of numerous stable and transient species [7-17], stimulated emission pumping of $\mathrm{C}_{3}$ and HCO using RFWM [18], and the application of a pulsed slit-jet expansion source to RFWM spectroscopy [19].

One area in which TC-RFWM spectroscopy has significant potential to contribute to our understanding is that of excited states that decay rapidly by intramolecular, nonradiative processes (for example, internal energy conversion and predissociation). These states can be difficult to study by traditional spectroscopic techniques, such as laser-induced fluorescence and resonantly enhanced multiphoton ionization. The recent application of TC-RFWM to studies of predissociating and autoionizing states in nitric oxide $[8,20]$ provide examples where this capability of TC-RFWM can be developed and tested. In these experiments, continuum states are accessed in the four-wave mixing process and the interpretation of the measured line profiles can provide important information on the dynamics of the decay processes. However, to obtain that information, a model of the expected line profile that takes into account the role of the continuum states in the four-wave mixing process is needed.

Diagrammatic perturbation theory has proven to be a very effective tool in analyzing the signals obtained in gas-phase, TC-RFWM spectroscopy. It has been used successfully by Williams and co-workers [21] to develop expressions for signal intensities observed in degenerate four-wave mixing [22], TC-RFWM for the case of stimulated emission pumping [18], and most recently they have reported general analytic signal expressions that can be applied to TC-RFWM experimental schemes used in double-resonance spectroscopy [21]. In this paper a similar approach is adopted to calculate the four-wave mixing signal that arises in cases where continuum states of the medium make contributions to the third-order susceptibility. In these cases, the rotatingwave approximation plays a key role and leads to distinct line-shape factors in the expression for the third-order polarization. To illustrate these effects, the line profile of a quasibound resonance is derived for several experimental implementations of TC-RFWM. It is shown that the TC-RFWM line profile for a quasibound resonance, modeled as a bound and continuum state coupled by configuration interactions, can be written in terms of the same line-shape parameters used in the analysis of linear spectra. A comparison shows that the quasibound line profile observed by using TCRFWM is significantly different from the line profile observed by using a linear spectroscopic technique and a proper 
treatment is required to obtain accurate decay parameters.

An analysis of the line profile of a quasibound resonance observed by using RFWM techniques has been reported previously by Wynne and co-workers in the context of thirdharmonic generation in alkali-metal atomic vapor [23]. Their experiment used a configuration where a two-photon transition between two bound states of the metal was excited, followed by single-photon absorption to the autoionizing level. The detected, third-harmonic signal arose from the downward transition from the autoionizing level to the lowest of the two bound states. In this case, the theoretical analysis of the observed spectra [24,25] involved the coupling between each of the two bound states and the autoionizing state and, as a consequence, required the introduction of two sets of line-shape parameters. The TC-RFWM schemes considered here, however, are distinct from this third-harmonic generation scheme and thus require a different analysis.

The paper is organized in the following way. In Sec. II, the configuration-interaction treatment of quasibound states is briefly reviewed and in Sec. III the implementation of this model in the derivation of the third-order electric susceptibility is presented. In Sec. IV the expected line-shape function for several TC-RFWM experimental configurations used in double-resonance spectroscopy is explicitly calculated. In Sec. V those line profiles are discussed and compared to their equivalents in linear spectroscopy. Finally, in Sec. VI the results are summarized.

\section{CONFIGURATION-INTERACTION TREATMENT OF QUASIBOUND STATES}

In the context of atomic and molecular spectroscopy, quasibound states arise from the interaction of discrete and continuum configurations of a system. Well-known examples include autoionization and predissociation. The mixed nature of quasibound states can result in asymmetric resonant structures in measurements of, for example, photoemission, photoabsorption, photodetachment, and photofragmentation reflecting the competing processes in the time evolution of the system. The observed line profiles hold important information about the decay dynamics of these resonances and a large number of different theoretical approaches have been devised to analyze them including the general and effective tool of multichannel quantum-defect theory (MQDT) [26]. The interaction of a single, isolated discrete level with a single continuum, however, can be treated more simply by using the configuration-interaction method of Fano $[27,28]$ and this is the model we use below to illustrate the role of continuum states in the calculation of TC-RFWM signals.

The configuration-interaction model is illustrated in Fig. 1(a) for the example of an autoionizing state. The state $|A\rangle$, well separated from any other discrete state, lies above the first ionization potential (IP) of the field-free Hamiltonian $H$ and autoionizes with a rate $\Gamma$. Because of the degeneracy in energy between $|A\rangle$ and the ionization continuum, neither $|A\rangle$ nor the unperturbed continuum is an exact eigenstate of $H$. By diagonalizing the portion of the energy matrix that belongs to the set of states above the IP, including $|A\rangle$ and the unperturbed continuum, a new set of eigenstates that form a continuous manifold of so-called Fano states is ob- (a)

(b)
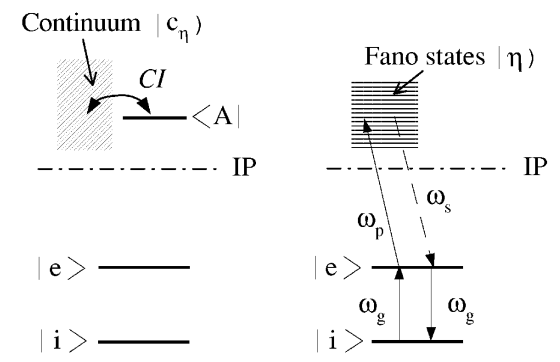

FIG. 1. (a) Configuration-interaction (CI) model of a quasibound resonance. (b) Nonparametric TC-RFWM process discussed in the text.

tained. A Fano state is indicated by $\mid \eta$ ), where the reduced index $\eta$ is defined as

$$
\eta=\frac{\omega_{\eta e}-\omega_{R}}{\Gamma / 2} .
$$

Here $\hbar \omega_{\eta e}$ is the energy difference between the Fano state and the intermediate state $|e\rangle$ and $\hbar \omega_{R}$ corresponds to the energy difference between the original autoionizing state $|A\rangle$ and $|e\rangle$ plus a small energy shift factor that depends on $\Gamma$ and is often negligible [29]. The energy index $\eta$ takes on numerical values within a suitable continuous interval $\mathcal{D}_{\eta}$ that spans the set of Fano states.

The spectral density of the dipole transition probability for photoexcitation from $|e\rangle \rightarrow \mid \eta)$ is given by [29]

$$
\left|\left\langle e\left|\mu_{\nu}\right| \eta\right)\right|^{2}=p_{c} \frac{(q+\eta)^{2}}{1+\eta^{2}}+p_{u} .
$$

The right-hand side of Eq. (2) represents the well-known Fano line profile of an isolated autoionizing resonance measured using a linear spectroscopic technique such as photoabsorption. Here $p_{c}$ is the direct photoionization probability, namely, $\left|\left\langle c_{\eta}\left|\mu_{\nu}\right| e\right\rangle\right|^{2}$, where $\left|c_{\eta}\right\rangle$ is the unperturbed continuum state having the same energy of the Fano state $\mid \eta$ ) and $p_{u}$ is the probability of transitions to other ionization continua not interacting with the autoionizing state. The $q$ asymmetry parameter in Eq. (2) is given by

$$
q=\frac{\left\langle A\left|\mu_{\nu}\right| e\right\rangle}{\pi\left\langle A|H| c_{\eta}\right\rangle\left\langle c_{\eta}\left|\mu_{\nu}\right| e\right\rangle},
$$

while the lifetime of the autoionizing level is given by $\hbar \Gamma \simeq 2 \pi\left|\left\langle A|H| c_{\eta}\right\rangle\right|^{2}$. The values of $q, p_{c}, p_{u}$, and $\Gamma$ may be considered to be nearly $\eta$ independent for a range of values of $\eta$ about $\eta=0$ [29].

\section{FOUR-WAVE MIXING INVOLVING QUASIBOUND STATES}

\section{A. General considerations}

The signal intensity in a four-wave mixing (FWM) process is proportional to the squared modulus of the cycle average of the third-order polarization induced by the incident 
fields. The expression of the $\nu$ th Cartesian component of the polarization, at a given point $\mathbf{r}$ and time $t$, can be written in the general case as [6]

$$
\begin{aligned}
P_{\nu}^{(3)}(\mathbf{r}, t)= & \\
& \frac{1}{(2 \pi)^{3}} \sum_{\alpha_{3}, \alpha_{2}, \alpha_{1}=1}^{3} \int_{-\infty}^{\infty} d \omega_{3} \int_{-\infty}^{\infty} d \omega_{2} \int_{-\infty}^{\infty} d \omega_{1} \\
& \times \chi_{\nu \alpha_{3} \alpha_{2} \alpha_{1}}^{(3)} \mathfrak{E}_{\alpha_{3}}\left(\mathbf{r}, \omega_{3}\right) \mathfrak{E}_{\alpha_{2}}\left(\mathbf{r}, \omega_{2}\right) \\
& \times \mathfrak{E}_{\alpha_{1}}\left(\mathbf{r}, \omega_{1}\right) \exp \left(-i \omega_{s} t\right)
\end{aligned}
$$

The indices $\alpha_{3}, \alpha_{2}, \alpha_{1}$ refer to Cartesian components and the numerical subindices identify the time ordering: namely, $\mathfrak{E}_{\alpha_{m}}\left(\mathbf{r}, \omega_{m}\right)$ is the Fourier transform of the $\alpha_{m}$ component of $m$ th field interacting with the medium. The signal frequency $\omega_{s}$ equals $\omega_{1}+\omega_{2}+\omega_{3}$ and $\chi_{\nu \alpha_{3} \alpha_{2} \alpha_{1}}^{(3)}$ is the third-order electric susceptibility tensor.

The expression for $\chi_{\nu \alpha_{3} \alpha_{2} \alpha_{1}}^{(3)}$ as a function of $\omega_{1}, \omega_{2}, \omega_{3}$ may be obtained by solving, to the third perturbative order in the interaction with external fields, the von Neumann equation that describes the time evolution of the material density operator. This procedure is described in Ref. [30] and will not be recalled here. We adapt the general expression of the susceptibility to the situation shown in Fig. 1(a) by making the assumption that the thermal equilibrium population of all states is negligible except for the lowest state $|i\rangle$ and by observing that the relevant matter states entering the evaluation of $\chi^{(3)}$ include two discrete states and the continuous manifold of Fano states. The susceptibility can then be expressed as

$$
\begin{aligned}
\chi_{\nu \alpha_{3} \alpha_{2} \alpha_{1}}^{(3)}= & -\frac{\rho_{i i}^{0} N}{6 \hbar^{3}} \sum_{p e r m} \int_{\mathcal{D}_{\eta}} d \eta \sum_{a, b, c=i, e, \eta} \mu_{\nu}^{i a} \mu_{\alpha_{3}}^{a b} \mu_{\alpha_{2}}^{b c} \mu_{\alpha_{1}}^{c i} \\
& \times\left[\mathcal{G}^{c b}\left(\omega_{3}+\omega_{2}+\omega_{1}\right) \mathcal{G}^{c a}\left(\omega_{2}+\omega_{1}\right) \mathcal{G}^{c i}\left(\omega_{1}\right)\right. \\
& +\mathcal{G}^{c b}\left(\omega_{3}+\omega_{2}+\omega_{1}\right) \mathcal{G}^{c a}\left(\omega_{2}+\omega_{1}\right) \mathcal{G}^{i a}\left(\omega_{1}\right) \\
& +\mathcal{G}^{c b}\left(\omega_{3}+\omega_{2}+\omega_{1}\right) \mathcal{G}^{i b}\left(\omega_{2}+\omega_{1}\right) \mathcal{G}^{i a}\left(\omega_{1}\right) \\
& +\mathcal{G}^{a i}\left(\omega_{3}+\omega_{2}+\omega_{1}\right) \mathcal{G}^{b i}\left(\omega_{2}+\omega_{1}\right) \mathcal{G}^{c i}\left(\omega_{1}\right) \\
& -\mathcal{G}^{c b}\left(\omega_{3}+\omega_{2}+\omega_{1}\right) \mathcal{G}^{d b}\left(\omega_{2}+\omega_{1}\right) \mathcal{G}^{i a}\left(\omega_{1}\right) \\
& -\mathcal{G}^{b a}\left(\omega_{3}+\omega_{2}+\omega_{1}\right) \mathcal{G}^{c a}\left(\omega_{2}+\omega_{1}\right) \mathcal{G}^{c i}\left(\omega_{1}\right) \\
& -\mathcal{G}^{b a}\left(\omega_{3}+\omega_{2}+\omega_{1}\right) \mathcal{G}^{b i}\left(\omega_{2}+\omega_{1}\right) \mathcal{G}^{c i}\left(\omega_{1}\right) \\
& \left.-\mathcal{G}^{i c}\left(\omega_{3}+\omega_{2}+\omega_{1}\right) \mathcal{G}^{i b}\left(\omega_{2}+\omega_{1}\right) \mathcal{G}^{i a}\left(\omega_{1}\right)\right]
\end{aligned}
$$

where $\rho_{i i}^{0}$ is the diagonal element of the material density matrix corresponding to the thermal-equilibrium population of the state $|i\rangle$ and $N$ the number density of the medium. The first summation accounts for the six possible permutations of $\omega_{1}, \omega_{2}$, and $\omega_{3}$, while $a, b$, and $c$ in the second summation are matter state indices that may take as values $i, e$, or $\eta$. The integration over $\eta$ represents the summation over the set of Fano states as required by the definition of $\chi^{(3)}$.
In addition, the following abbreviation for the four-point dipole matrix element has been adopted:

$$
\mu_{\nu}^{i a} \mu_{\alpha_{3}}^{a b} \mu_{\alpha_{2}}^{b c} \mu_{\alpha_{1}}^{c i}=\left\langle i\left|\mu_{\nu}\right| a\right\rangle\left\langle a\left|\mu_{\alpha_{3}}\right| b\right\rangle\left\langle b\left|\mu_{\alpha_{2}}\right| c\right\rangle\left\langle c\left|\mu_{\alpha_{1}}\right| i\right\rangle .
$$

In the square brackets are eight triplets of formal Green propagators [30]. In the weak-field limit where no saturation effects occur and assuming no velocity effects, each Green propagator in Eq. (5) is given by

$$
\mathcal{G}^{a b}\left(\omega_{\mathfrak{E}}\right)=\frac{1}{\omega_{\mathfrak{E}}-\omega_{a b}+i \Gamma_{a b}}
$$

and is a function of a field-related frequency $\omega_{\mathfrak{E}}$, which can take on the values $\omega_{1}, \omega_{2}+\omega_{1}$, or $\omega_{3}+\omega_{2}+\omega_{1}$. The Green propagators depend parametrically upon an intrinsic frequency $\omega_{a b}$, where $\omega_{a b}=-\omega_{b a}$ and $\hbar\left|\omega_{a b}\right|$ is the energy gap between states $|a\rangle$ and $|b\rangle$, and on $\Gamma_{a b}$, which is a relaxation factor. The relaxation factor $\Gamma_{a b}$ is regarded simply as the average decay rate of states $|a\rangle$ and $|b\rangle$ because there is no collisional pure dephasing of the coherence between states in the low-density environments considered here $[18,21]$. The decay rate of any Fano state is assumed to be $\Gamma$.

\section{B. Incident fields}

The TC-RFWM schemes can often be interpreted in terms of a conceptually simple picture of the formation and the scattering from laser-induced gratings. Two nearly copropagating laser beams overlapped at a small crossing angle in a medium and resonant with a transition in that medium will produce a spatial modulation in the optical properties of the medium that constitutes a diffraction grating. A third laser beam is then scattered off this laser-induced grating and the scattered beam is detected as the four-wave mixing signal. The first two laser beams are called grating beams and the third beam is called the probe beam. The two excitation schemes to be considered are depicted in Figs. 1(b) and 2(a). For example, in the scheme shown in Fig. 1(b), the grating beams with frequency $\omega_{g}$ are tuned on the transition $|i\rangle \leftrightarrow|e\rangle$, and the third beam having the frequency $\omega_{p}$ is tuned to scan over transitions $|e\rangle \rightarrow \mid \eta)$. The scattered light beam detected as the signal is shown as a dotted downward arrow with a frequency $\omega_{s}=\omega_{p}$.

Assuming $\delta$-like laser bandwidths, the plane-wave approximation, and the same linear polarization for all laser beams, the Fourier-transformed fields in Eq. (4) may be written as

$$
\begin{aligned}
\mathfrak{E}_{\alpha_{1}}\left(\mathbf{r}, \omega_{1}\right)= & \mathcal{E}^{g} \mathbf{e}_{\alpha_{1}} \delta\left(\omega_{1}-\omega_{g}\right) \exp \left(i \mathbf{k}_{g_{1}} \cdot \mathbf{r}\right) \\
& +\mathcal{E}^{g *} \mathbf{e}_{\alpha_{1}} \delta\left(\omega_{1}+\omega_{g}\right) \exp \left(-i \mathbf{k}_{g_{1}} \cdot \mathbf{r}\right), \\
\mathfrak{E}_{\alpha_{2}}\left(\mathbf{r}, \omega_{2}\right)= & \mathcal{E}^{g} \mathbf{e}_{\alpha_{2}} \delta\left(\omega_{2}-\omega_{g}\right) \exp \left(i \mathbf{k}_{g_{2}} \cdot \mathbf{r}\right) \\
& +\mathcal{E}^{g *} \mathbf{e}_{\alpha_{2}} \delta\left(\omega_{1}+\omega_{g}\right) \exp \left(-i \mathbf{k}_{g_{2}} \cdot \mathbf{r}\right), \\
\mathfrak{E}_{\alpha_{3}}\left(\mathbf{r}, \omega_{3}\right)= & \mathcal{E}^{p} \mathbf{e}_{\alpha_{3}} \delta\left(\omega_{3}-\omega_{p}\right) \exp \left(i \mathbf{k}_{p} \cdot \mathbf{r}\right) \\
& +\mathcal{E}^{p *} \mathbf{e}_{\alpha_{3}} \delta\left(\omega_{3}+\omega_{g}\right) \exp \left(-i \mathbf{k}_{p} \cdot \mathbf{r}\right)
\end{aligned}
$$


(a)

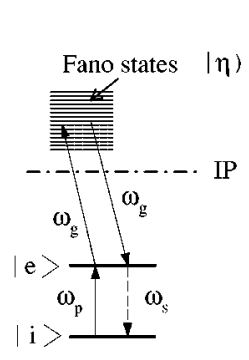

(b)

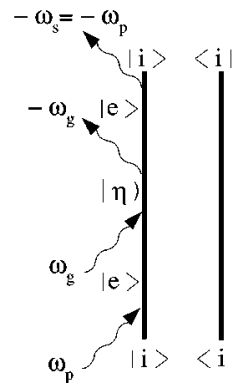

FIG. 2. (a) Parametric TC-RFWM scheme discussed in the text. (b) Corresponding double-sided Feynman diagrams for the scheme shown in (a).

In Eq. (7), $\mathcal{E}^{g}$ and $\mathcal{E}^{p}$ are the complex amplitudes of grating and probe beams, respectively, $\mathbf{k}_{g_{1}}$ and $\mathbf{k}_{g_{2}}\left(\left|\mathbf{k}_{g_{1}}\right|=\left|\mathbf{k}_{g_{2}}\right|\right.$ $\left.=\omega_{g} / c\right)$ are the wave vectors of the grating beams, $\mathbf{k}_{p}$ is the wave vector of the probe beam, and $\mathbf{e}$ is a real unit vector. Overall, any triple product $\mathfrak{E}_{\alpha_{3}}\left(\mathbf{r}, \omega_{3}\right) \mathfrak{E}_{\alpha_{2}}\left(\mathbf{r}, \omega_{2}\right) \mathfrak{E}_{\alpha_{1}}\left(\mathbf{r}, \omega_{1}\right)$ has eight terms. Substituting the three expressions for the fields written in the form of Eq. (7) into Eq. (4) for the polarization, the triple frequency integration can be immediately performed. Next, by choosing the phasematching condition as $\omega_{s}=\omega_{p}$, the number of terms in the triple product is reduced from eight to three, with Fourier transforms centered on $\omega_{g},-\omega_{g}$, and $\omega_{p}$, respectively, in any time-ordering permutation. Correspondingly, the argument $\omega_{\mathfrak{E}}$ of the Green propagator in Eq. (6) assumes only six values: $\omega_{\mathfrak{E}}=\omega_{p}, \pm \omega_{g}, \omega_{p} \pm \omega_{g}, 0$.

\section{Rotating-wave approximation}

The expression for the third-order susceptibility in Eq. (5) can be simplified by carefully applying the rotating-wave approximation (RWA) using the double-sided (DS) Feynman diagram formalism [31]. Consider the excitation scheme shown in Fig. 1(b) and the Feynman diagrams that describe it, depicted in Fig. 3. At thermal equilibrium the material density operator contains only the projector onto the lowest state, namely, $\rho_{i i}^{0}|i\rangle\langle i|$, and therefore the first interaction must be an absorption and resonance is obtained only if the frequency of the field is $\omega_{g}=\omega_{e i}$ (creation of the coherence $\rho_{e i}|e\rangle\langle i|$ ) or $\omega_{g}=-\omega_{e i}$ (creation of the coherence $\left.\rho_{i e}|i\rangle\langle e|\right)$. The interaction with the probe field can only take place when the coherence $\rho_{e i}|e\rangle\langle i|$ (or the population $\left.\rho_{e e}|e\rangle\langle e|\right)$ has been generated and therefore not before the interaction with the field at frequency $\omega_{g}$. That is, the temporal sequence $\omega_{p},-\omega_{g}$ is not allowed. The effect is the creation of a continuous manifold of coherences $\left.\rho_{\eta i} \mid \eta\right)\langle i|$ (or $\left.\rho_{\eta e} \mid \eta\right)\langle e|$ ) involving all Fano states. At the end of the three interactions, a signal at frequency $\omega_{p}$ is detected, that is, an emission takes place by a downward transition $\mid \eta) \rightarrow|e\rangle$ and a population $\rho_{e e}|e\rangle\langle e|$ is left in the medium. Only three resonant diagrams are consistent with this scheme and they are represented in Fig. 3. Each diagram is continuously replicated for every $\mid \eta)$. (a)

(b)

(c)

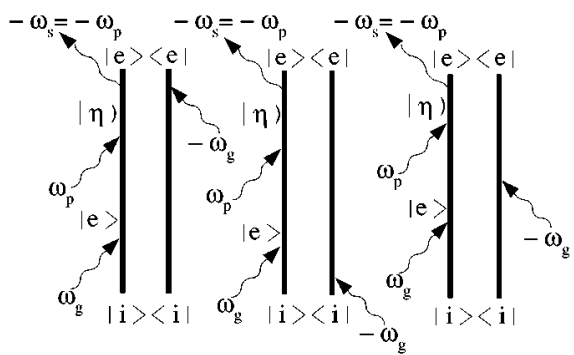

FIG. 3. Double-sided Feynman diagrams for the TC-RFWM scheme shown in Fig. 1.

To clarify the criterion underlying the choice of the diagrams in Fig. 3, the connection between the terms in Eq. (5) for the susceptibility and the diagrams is recalled [30]. Each diagram represents one of the triplets of Green propagators in Eq. (5) and a single propagator, associated with a specific interaction, is symbolized by a wavy line. The argument of each propagator is determined by adding the frequency that labels the corresponding wavy line to the frequencies of all wavy lines preceding it in chronological order, if any. The indices of intrinsic frequency and relaxation factor [see Eq. (6)] refer to the pair of states forming the coherence that is given by the considered interaction. Finally, the sign of the triplet is $(-1)^{n}$, where $n$ is the number of wavy lines acting from the right.

The choice of diagrams in Fig. 3 is equivalent to the selective application of the RWA to the expression for the third-order susceptibility, Eq. (5). When the argument of a propagator in Eq. (5) is $\omega_{\mathfrak{E}}=\omega_{p}, \pm \omega_{g}, \omega_{p}+\omega_{g}, 0$ and the intrinsic frequency is $\omega_{\eta e}, \mp \omega_{e i}, \omega_{\eta i}, 0$, respectively, a resonant term is obtained, that is, the frequencies in the denominator of the propagator cancel each other and only a relaxation factor remains whose order of magnitude is always in the radio-frequency range thereby producing an overall value for the propagator that is large and dominates in its contribution to the susceptibility. In any other case, a noncancelling optical frequency results in the denominator and the entire triplet to which that propagator belongs can be neglected as being much smaller in magnitude than the contribution due to resonant terms.

The application of the RWA to terms including the Fano states, however, does not simplify in the same manner and requires careful consideration. The nonvanishing terms will include in the summation each propagator with an argument $\omega_{p}$ (or $\omega_{p}+\omega_{g}$ ) for any value of $\eta$ in $\mathcal{D}_{\eta}$, that is, for any possible value of $\omega_{\eta e}\left(\right.$ or $\left.\omega_{\eta i}\right)$. This is justified from a physical point of view. As $\eta$ scans continuously over $\mathcal{D}_{\eta}$, selecting different Fano states, the corresponding detuning values $\omega_{p}-\omega_{\eta e}$ (or $\omega_{p}+\omega_{g}-\omega_{\eta i}$ ), at a fixed $\omega_{p}$ (or at a fixed $\omega_{p}$ and $\omega_{g}$ ) fall in a frequency interval typically ranging from 0 to $\Gamma$, which is the characteristic width of the Fano state distribution as given by Eqs. (1) and (2). Thus we have two types of nonvanishing terms. In the purely resonant case, that is, when the detuning is 0 , the denominator of the corre- 
sponding Green propagator will consist again only of a relaxation factor $\Gamma_{\eta e}\left(\Gamma_{\eta i}\right)$. In the case where the detuning is of the order of $\Gamma$, we obtain a denominator of the type $\Gamma+i \Gamma_{\eta e}\left(\right.$ or $\left.+i \Gamma_{\eta i}\right)$, which is of the same order of magnitude as the denominator for the zero-detuning case. In fact, $\Gamma$ dominates over the decay rate of bound states and therefore the relationship

$$
\Gamma_{\eta i} \simeq \Gamma_{\eta e} \simeq \Gamma / 2
$$

holds.

The derivation from first principles of the expression for the susceptibility involving a quasibound resonance is useful because it highlights that the entire manifold of Fano states contributes to the susceptibility. At first sight, the Feynman diagrams that describe this process are the same diagrams that would be found by considering a level scheme of three discrete states, roughly repeated as many times as there are Fano states. However, it is ambiguous to borrow, a priori, the DS Feynman diagrams that apply to the case of discrete states and somehow coherently add these diagrams to get a sense of the effect of the continuum. The risk is to argue that the range of continuum states to be included depends on the laser bandwidth. The presentation here, however, demonstrates that this would not be correct. No coherent excitation of states takes place in the model because the laser bandwidth is regarded as simply $\delta$-like, yet all the Fano states contribute to the susceptibility and thus to the TC-RFWM signal.

This is a direct result of the general definition of the susceptibility combined with the proper application of the RWA. According to the general definition given in Ref. [30] and substantially recalled here in Eq. (5), the susceptibility is a property inherent to the medium, depending only on the distribution of stationary states (belonging to both discrete and continuum configurations) of the medium itself and does not involve features of the external field. In addition, the application of the RWA to the susceptibility uses only the center-line frequency of the fields, with no consideration of their bandwidth. Overall, therefore, the entire evaluation of the susceptibility carried out here does not depend on the laser bandwidth. It is rather the third-order polarization that exhibits such a dependence as explicitly shown by Eq. (4) in which the Fourier transform of the input fields is directly involved. Apart from practical consequences concerning calculations and numerical results, this conceptual aspect of treating continuum states is important and emphasized here because it is strictly correlated with the nonlinear nature of the technique and represents an important difference from conventional linear spectroscopy.

\section{RESULTS}

\section{A. Nonparametric process}

In standard, nonlinear optics terminology, a FWM process described by DS Feynman diagrams in which interactions occur on both sides is called nonparametric and this conventional label is adopted for the processes shown in Figs. 1(b) and 3. Carrying out the prescriptions discussed above for this excitation scheme, Eq. (5) for the susceptibility reduces to

$$
\chi_{\nu \alpha_{3} \alpha_{2} \alpha_{1}}^{(3)}=\frac{\rho_{i i}^{0} N}{6 \hbar^{3}} \int_{\mathcal{D}_{\eta}} \mu_{\nu}^{i e} \mu_{\alpha_{3}}^{e \eta} \mu_{\alpha_{2}}^{\eta e} \mu_{\alpha_{1}}^{e i} \mathfrak{L}\left(\omega_{g}, \omega_{p} ; \eta\right) d \eta
$$

where the (complex) line-shape function $\mathfrak{L}\left(\omega_{g}, \omega_{p} ; \eta\right)$ has been introduced as

$$
\begin{aligned}
\mathfrak{L}\left(\omega_{g}, \omega_{p} ; \eta\right)= & \frac{1}{\omega_{p}-\omega_{\eta e}+i \Gamma_{\eta e}} \\
& \times\left[\frac{1}{i \Gamma_{e e}\left(\omega_{g}-\omega_{e i}+i \Gamma_{e i}\right)}\right. \\
& +\frac{1}{i \Gamma_{e e}\left(-\omega_{g}+\omega_{e i}+i \Gamma_{e i}\right)} \\
& \left.+\frac{1}{\left(\omega_{g}-\omega_{e i}+i \Gamma_{e i}\right)\left(\omega_{p}+\omega_{g}-\omega_{\eta i}+i \Gamma_{\eta i}\right)}\right] .
\end{aligned}
$$

Equation (10) for the line-shape function may be simplified by observing that

$$
\omega_{\eta i}=\omega_{\eta e}+\omega_{e i}
$$

and by assuming that the grating beams are in resonance, that is, $\omega_{g}=\omega_{e i}$. Also, in the limit of an infinitely long-lived ground state, the following two approximations hold [18]:

$$
\begin{gathered}
\Gamma_{\eta e} \simeq \Gamma_{e i}+\Gamma_{\eta i} \\
\Gamma_{e e} \simeq 2 \Gamma_{e i} .
\end{gathered}
$$

Substituting these relations into Eq. (10) yields a more compact line-shape function

$$
\mathfrak{L}\left(\omega_{p} ; \eta\right)=\frac{1}{\Gamma_{e i}^{2}\left(\omega_{p}-\omega_{\eta e}+i \Gamma_{\eta i}\right)} .
$$

By assuming further that the polarizations of the input fields are all orientated along the $\nu$ th axis in the reference frame of the laboratory, the overall polarization can be written as

$$
\begin{aligned}
P_{\nu}^{(3)}(\mathbf{r}, t)= & \frac{\rho_{i i}^{0} N}{6 h^{3}} \mathcal{E}^{p}\left|\mathcal{E}^{g}\right|^{2}\left|\left\langle i\left|\mu_{\nu}\right| e\right\rangle\right|^{2} \exp \left[-i\left(\omega_{p} t+\mathbf{k}_{s} \cdot \mathbf{r}\right)\right] \\
& \times \int_{\mathcal{D}_{\eta}}\left|\left\langle e\left|\mu_{\nu}\right| \eta\right)\right|^{2} \mathfrak{L}\left(\omega_{g}, \omega_{p} ; \eta\right) d \eta
\end{aligned}
$$

where $\left|\mathbf{k}_{s}\right|$ satisfies the phase-matching condition

$$
\left|\mathbf{k}_{s}\right|=\left|\mathbf{k}_{g_{1}}-\mathbf{k}_{g_{2}}+\mathbf{k}_{p}\right|=\omega_{p} / c .
$$

An example geometry, often referred to as the laser-induced grating configuration [17] and used by many experimentalists, is illustrated in Fig. 4. The direction of $\mathbf{k}_{s}$ is derived in Appendix A.

Frequently, in TC-RFWM experiments that use pulsed lasers, probe light pulses can be either overlapped or delayed in time with respect to the grating beam light pulses. In the case of a delayed probe pulse, the diagram shown in Fig. 3(a) 


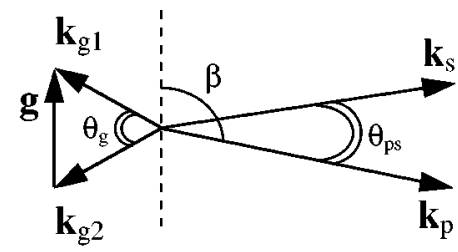

FIG. 4. Wave-vector diagram illustrating an example phasematching condition for the TC-RFWM process.

has no physical meaning. As a consequence, the line-shape function for the case of a delayed probe, obtained under the same assumptions used to derive Eq. (13), is given by

$$
\mathfrak{L}\left(\omega_{p} ; \eta\right)=\frac{1}{\Gamma_{e i}^{2}\left(\omega_{p}-\omega_{\eta e}+i \Gamma_{\eta e}\right)}
$$

An expression for the intensity of the TC-RFWM signal can now be obtained by introducing the reduced frequency

$$
\xi_{p} \equiv \frac{\omega_{p}-\omega_{R}}{\Gamma / 2}
$$

and substituting Eq. (2) for the transition probability to the Fano states into the equation (14) for the polarization and taking the absolute square. The same final expression is obtained for both line-shape functions given in Eqs. (13) and (16). In this expression for the TC-RFWM signal, the factor relevant to the line profile is given by

$$
\Im\left(\xi_{p}\right) \propto\left|\frac{1}{\Gamma_{e i}^{2} \Gamma} \int_{\mathcal{D}_{\eta}} d \eta \frac{1}{\xi_{p}-\eta+i}\left[p_{c} \frac{(q+\eta)^{2}}{1+\eta^{2}}+p_{u}\right]\right|^{2} .
$$

By using the integration procedure illustrated in the Appendix $\mathrm{B}$, the line profile can be written, to within a constant coefficient, as

$$
\Im\left(\xi_{p}\right)=\frac{\left(q^{2}+2 r+1\right)^{2}+\left[2 q+(1+r) \xi_{p}\right]^{2}}{\xi_{p}^{2}+4},
$$

where $r \equiv p_{u} / p_{c}$. Before proceeding with a discussion of this line profile, the analogous line profile for the mixing scheme shown in Fig. 2 is derived.

\section{B. Parametric process}

In Fig. 2(a) a second excitation scheme used to advantage in TC-FWM experiments is shown. Here the grating beams are in resonance with the upper transition and the probe is tuned to the lower transition. The detected signal then arises from the bound-to-bound transition $|e\rangle \rightarrow|i\rangle$. Based upon the same principles illustrated for the previous case, only one DS Feynman diagram, shown in Fig. 2(b), is involved in the mixing process. In this diagram all interactions are on the same side and thus the process is labeled as parametric. Following the same prescriptions used above, the line-shape function for this process is found to be

$$
\mathfrak{L}\left(\omega_{g}, \omega_{p} ; \eta\right)=\frac{1}{\left(\omega_{p}-\omega_{e i}+i \Gamma_{e i}\right)^{2}\left(\omega_{p}+\omega_{g}-\omega_{\eta i}+i \Gamma_{\eta i}\right)} .
$$

By using Eqs. (8) and (11) and introducing the reduced variables

$$
\begin{gathered}
\xi_{g}=\frac{\omega_{g}-\omega_{R}}{\Gamma / 2}, \\
d_{p}=\frac{\omega_{p}-\omega_{e i}}{\Gamma_{e i}},
\end{gathered}
$$

the line profile factor in the expression for the signal intensity is given by

$$
\begin{aligned}
\Im\left(\xi_{g}, d_{p}\right) \propto & \mid \frac{1}{\left(d_{p}+i\right)^{2}} \int_{\mathcal{D}_{\eta}} d \eta \frac{1}{d_{p}+\xi_{g}-\eta+i} \\
& \times\left.\left[p_{c} \frac{(q+\eta)^{2}}{1+\eta^{2}}+p_{u}\right]\right|^{2} .
\end{aligned}
$$

By using again the integration procedure illustrated in Appendix $\mathrm{B}$, the line profile in this case is given, to within a constant coefficient, by

$$
\begin{aligned}
\Im\left(\xi_{g}, d_{p}\right)= & \frac{1}{\left(d_{p}^{2}-1\right)^{2}+4 d_{p}^{2}} \\
& \times \frac{\left(q^{2}+2 r+1\right)^{2}+\left[2 q+(1+r)\left(\xi_{g}+d_{p}\right)\right]^{2}}{\left(\xi_{g}+d_{p}\right)^{2}+4} .
\end{aligned}
$$

\section{DISCUSSION}

\section{A. Nonparametric process}

In Fig. 5(a) the line profile given by Eq. (18) has been plotted in units of $\Gamma / 2$ for different values of the coefficient $q$. A suitable normalization has been adopted in all of the profiles shown in Fig. 5 and the profiles shown in the following figures in order to make comparisons of the different line profiles. Note that the Fano profile described by Eq. (2) and the profiles described by Eqs. (18) and (22) are nonnormalizable on a large energy scale since they tend to 1 far from resonance, which implies that their integral over the entire range of detuning will diverge. This behavior can be traced back to the assumption that the parameters involved in Eq. (2) are nearly energy independent. The profiles are therefore normalized by the area under the curves calculated on an abscissa interval of -10 to 10 . The difference introduced by this normalization compared to a normalization extending over all detunings will be small as long as the real behavior of the line profiles is to go to zero far away from resonance, which is the expected behavior as required for the total transition probability to be unitary.

It is immediately evident from Fig. 5(a) that the TCRFWM line profiles show an asymmetry degree that depends on $q$. The physical parameter $q$ is an important objective of spectroscopic investigations concerning quasibound states 

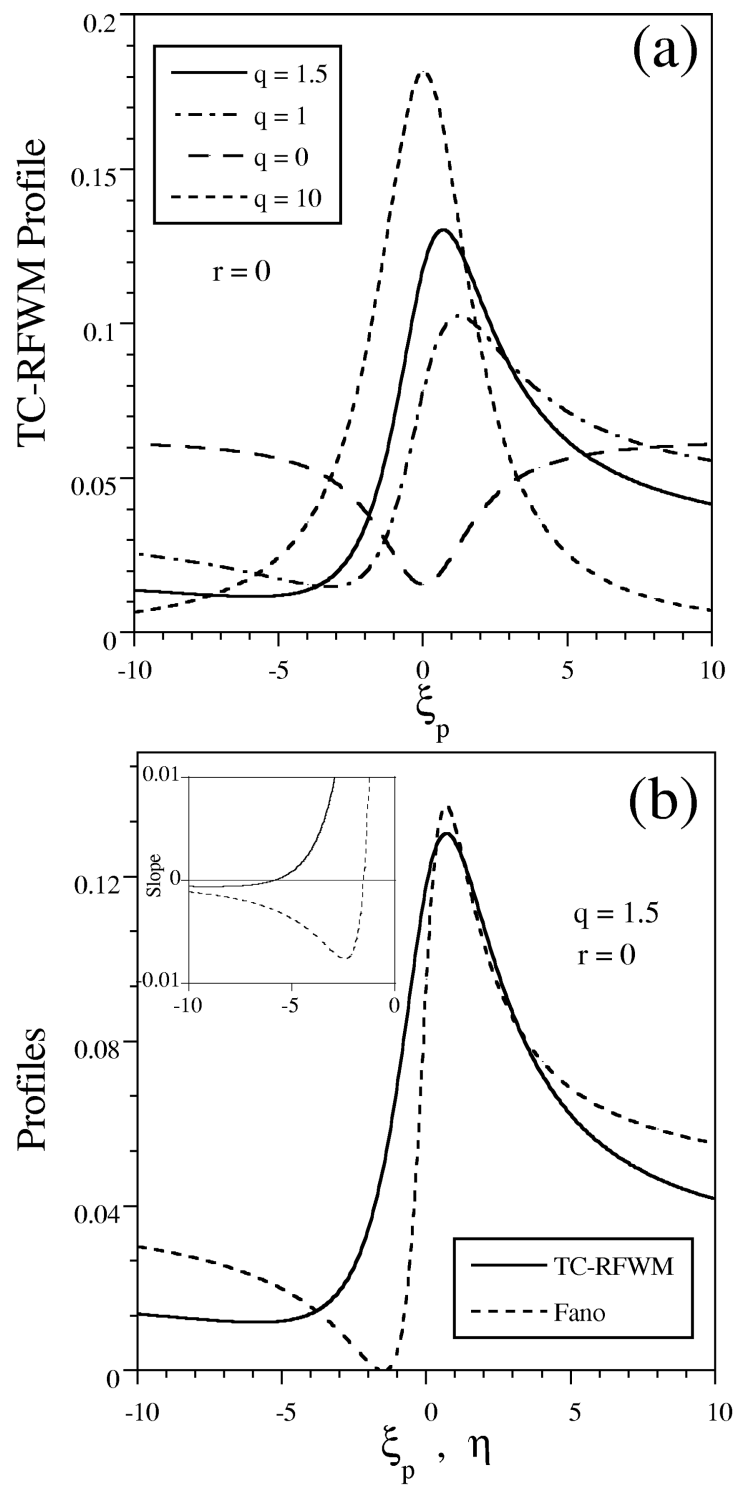

FIG. 5. (a) TC-RFWM profiles for different values of $q$ for the nonparametric TC-RFWM process depicted in Figs. 1(b) and 3. Profiles for negative values of $q$ are obtained by reversing the abscissa scale. (b) Comparison of a TC-RFWM profile and a Fano profile with $q=1.5$. Inset: slope of the two profiles in (b) for the detuning interval $(-10,0)$.

and may be extracted from experimental data through a fitting procedure based on Eq. (18). Thus TC-RFWM can be used effectively to obtain the same information made available by linear photoabsorption techniques. Further, the line profile given by Eq. (18) exhibits invariance under simultaneous $q$ and $\xi_{p}$ reversal expressed by

$$
\Im\left(\xi_{p} ; q\right)=\Im\left(-\xi_{p} ;-q\right) .
$$

The line profiles with opposite $q$ mirror each other about the axis $\xi_{p}=0$, which is also a characteristic of the Fano profiles described by Eq. (2). (See Appendix B for details.)

In Fig. 5(b) the line profile given by Eq. (18), for $q=1.5$ and $r=0$, is plotted along with the corresponding Fano line profile given by Eq. (2). Unlike the Fano profile, which exibits a zero at $\eta=-q$ for every value of $q$, the spectral line profile in Eq. (18) has no zero. In fact, the numerator consists of the sum of two squares that vanishes if and only if both terms vanish, but the first term $\left(q^{2}+2 r+1\right)^{2}$ is strictly positive for any value of $q$ (and $r$ ). Such a natural background is physically expected as evidence of the nonlinear nature of the process exhibited in these line profiles. Indeed, the response of the medium is always determined by the entire set of Fano states as discussed in Sec. III, which implies that, at any value of $\xi_{p}$, there are susceptibility terms containing non-vanishing dipoles of the form $\left|\left\langle e\left|\mu_{\nu}\right| \eta\right)\right|^{2}$, with $\eta \neq q$, which give a nonzero contribution to the signal. The resulting difference in the line profile is particularly evident in the inset in Fig. 5(b) showing the slope of the profiles in the range of detuning of interest. In addition to the separation between the slope zeros corresponding to the profile minima, the slope of the Fano profile goes through a minimum close to the zero of the profile. This peculiarity (resulting in a local inversion of curvature in the corresponding line profile) is absent in the TC-RFWM signal and causes the pedestals of the two line profiles to differ substantially. This behavior is a feature that is of crucial importance in analyzing observed line profiles and may represent, if experimentally confirmed, an important test of the model presented here.

While the sharp distinction in the shape of the two profiles tends to be reduced in the limit of very small and very large $q$, the above-mentioned natural background of the TCRFWM line shape remains even when $q=0$. This is in contrast to the Fano profile which can exhibit a so-called window resonance that reaches zero at its center. As shown in Fig. 5(a), the TC-RFWM window resonance does not reach the zero level, even when $r=0$. For large values of $q$, both line profiles given by Eqs. (2) and (18) tend to Lorentzian profiles with the following asymptotic behavior:

$$
\begin{gathered}
\frac{(q+\eta)^{2}}{1+\eta^{2}} \sim \frac{1}{\eta^{2}+1} \quad(\text { Fano }), \\
\Im\left(\xi_{p}\right) \sim \frac{1}{\xi_{p}^{2}+4} \quad(\text { TC-RFWM }) .
\end{gathered}
$$

The full width at half maximum (FWHM) of the Lorentzian in Eq. (24a) is 2 in units of $\Gamma / 2$ (or $\Gamma$ in unreduced units), while the FWHM extracted from Eq. (24b) is 4 in units of $\Gamma / 2$ (or $2 \Gamma$ in unreduced units). This is another important detail from an experimental point of view. Even though both profiles are asymptotically Lorentzian for large $q$, the physical value of $\Gamma$ is given by the FWHM when the autoionizing feature is probed by a linear photoabsorption technique, but it is given by the half-width at half maximum when it is probed by means of TC RFWM. In general, the difference in the denominators on the right-hand sides of Eqs. (2) and (18) will make the width of the TC-RFWM profile larger, for a given value of $q$, than the width of the corresponding Fano profile.

In Fig. 6, TC-RFWM line profiles from Eq. (18) are shown for different values of $r \equiv p_{u} / p_{c}$. For $q \approx 0$ and $|q| \gg 1$, increasing $r$ simply results in rescaling the entire profile with respect to the case of negligible $r$. The same aspect is observed in Fano profiles at any value of $q$ as shown in Fig. 7(b). A distinction does occur between the two 


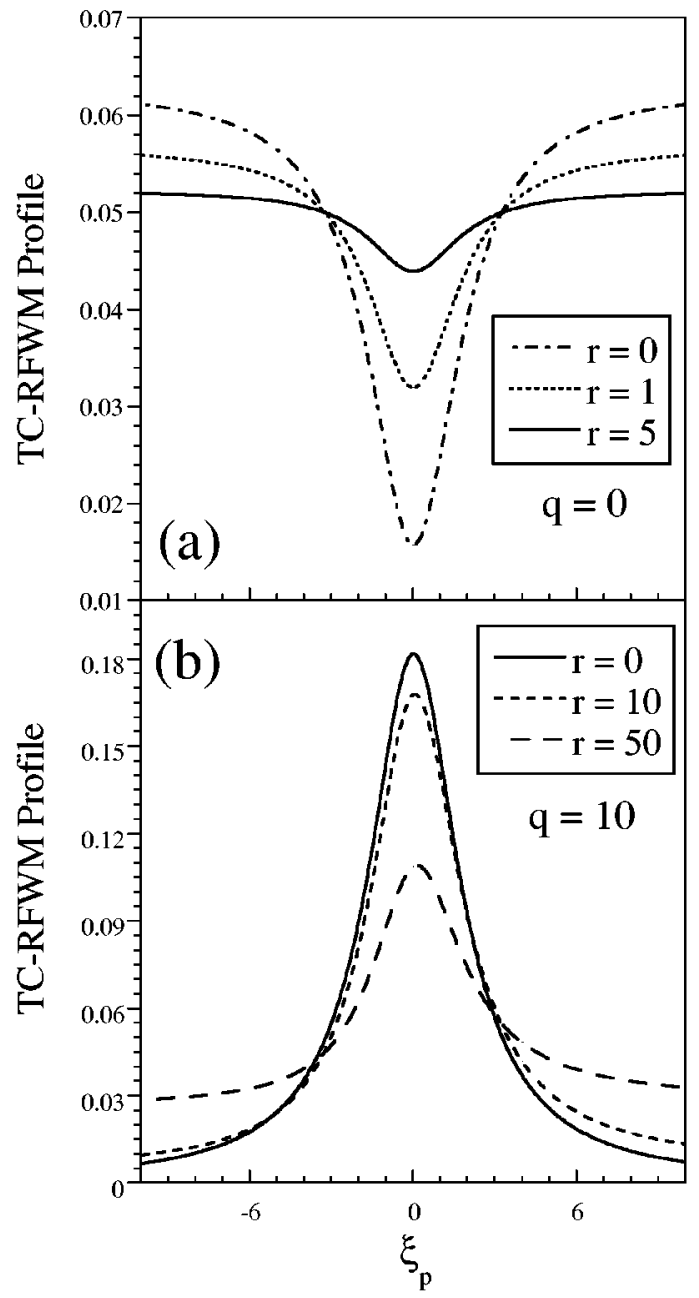

FIG. 6. TC-RFWM profiles for the nonparametric process depicted in Fig. 2 for several values of $r \equiv p_{u} / p_{c}$. (a) $q=0$ and (b) $q=10$.

cases, however, for $|q| \approx 1$. Here the interplay between $r$ and $q$, which is determined by the particular functional dependence upon these parameters that characterizes the line profile in Eq. (18), gives rise to a geometrical deformation of the TC-RFWM profile, which is indicated by the arrow in Fig. 7(a). Thus the asymmetry of the TC-RFWM profile is influenced by both $q$ and $r$. This is in contrast to the behavior of the Fano line shape shown in Fig. 7(b) whose asymmetry depends only on $q$.

\section{B. Parametric process}

The first result to note about the parametric process is that, in the case of negligible probe detuning $\left(d_{p} \simeq 0\right)$, the line profile given by Eq. (22) becomes that of Eq. (18) with $\xi_{g}$ in place of $\xi_{p}$. This implies that by scanning the grating beam frequency over the Fano states and detecting the coherent beam that emerges from the downward transition $|e\rangle \rightarrow|i\rangle$, profiles identical to those shown in Fig. 5(a) for the nonparametric process are obtained.

In this scheme, one can also fix the grating beam frequency and scan the probe laser to obtain a TC-RFWM spectrum. This is the case shown in Fig. 8. The profiles shown are obtained by scanning $d_{p}$ though zero, that is, by scanning
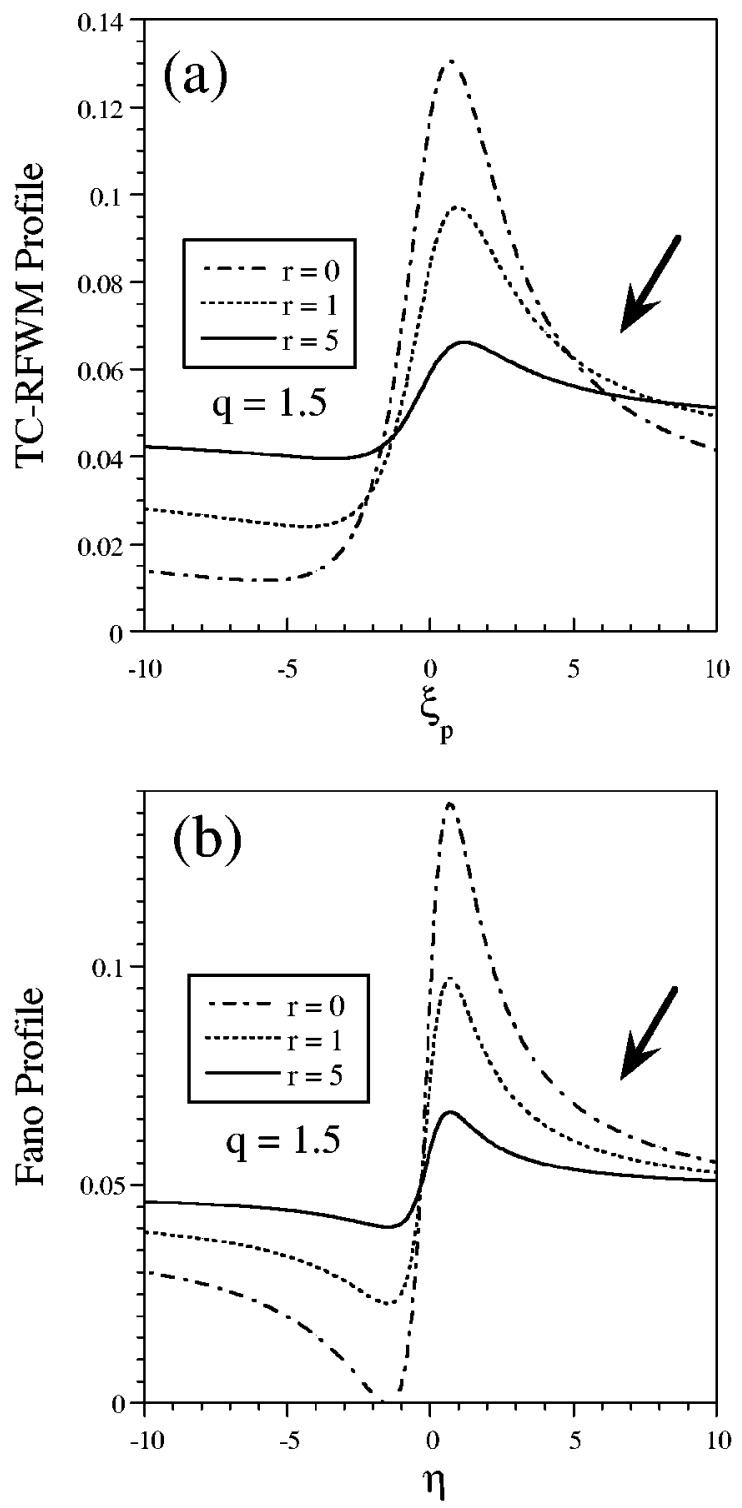

FIG. 7. Comparison of (a) TC-RFWM profiles for the nonparametric process and (b) Fano profiles with fixed $q=1.5$ and different values of $r \equiv p_{u} / p_{c}$.

$\omega_{p}$ across $\omega_{e i}$, for different values of $\xi_{g}$, which in this case is treated as an experimental parameter. Line profiles for $r=0, q=0$, and $q=1.5$ are shown. Although the detected signal arises from a bound-to-bound transition, because of the presence of quasibound states in the overall FWM process, the profile is non-Lorentzian for all values of $\xi_{g}, q$, and $r$. This behavior is in contrast to the TC-RFWM line shapes involving only bound states, which can be shown to be Lorentzian, as discussed by Williams and co-workers [21]. The profiles in Fig. 8 are also unlike the line profiles that would be observed by directly probing the transition $|e\rangle \rightarrow|i\rangle$ by means of a linear technique.

Both sets of profiles in Fig. 8 show the effect of tuning the grating beams to different frequencies within the manifold of Fano states. The effect consists of both a shift in the peak energy of the profile and a variation of the profile width. In the case of $q=0$, for example, the line profile in Eq. (22) can be written as 


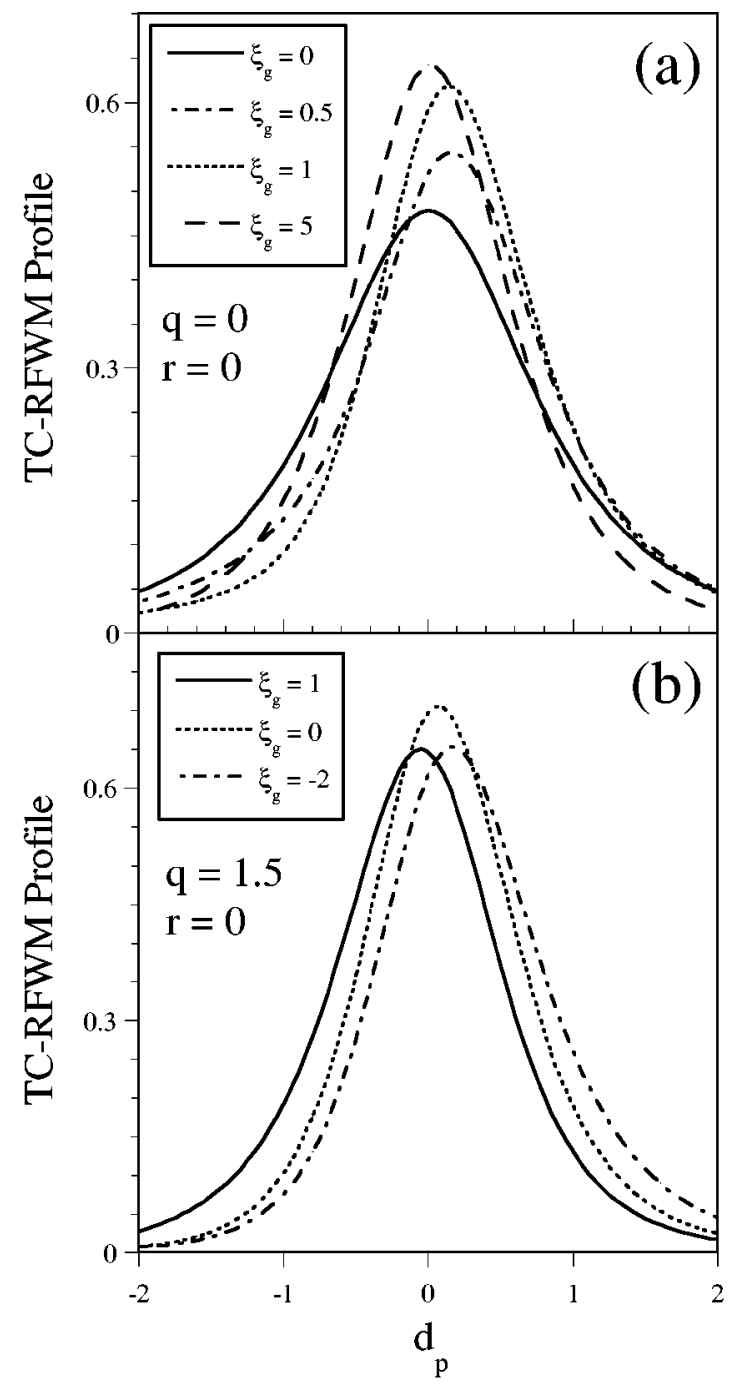

FIG. 8. TC-RFWM profiles for the parametric process depicted in Fig. 2 obtained by scanning the probe detuning $d_{p}$ for different values of grating detuning $\xi_{p}$. (a) $q=0$ and (b) $q=1.5$.

$$
\Im\left(d_{p} ; \xi_{g}\right)=\frac{1}{\left(d_{p}^{2}-1\right)^{2}+4 d_{p}^{2}} \frac{1+\left(\xi_{g}+d_{p}\right)^{2}}{\left(\xi_{g}+d_{p}\right)^{2}+4} .
$$

According to Eq. (25), both for $\xi_{g}=0$ and asymptotically for $\left|\xi_{g}\right| \gg 1, \Im\left(d_{p} ; \xi_{g}\right)$ is an even function of $d_{p}$. For $\xi_{g}=0$, where the process is still resonant, the following nonLorentzian profile is obtained:

$$
\Im\left(d_{p} ; \xi_{g}=0\right)=\frac{1}{\left(d_{p}^{2}-1\right)^{2}+4 d_{p}^{2}} \frac{1+d_{p}^{2}}{d_{p}^{2}+4} .
$$

For other values of the parameter $\xi_{g}$, the resulting profile has no symmetry with respect to $d_{p}$ because, as is evident in Eq. (25), the expression for $\Im\left(d_{p} ; \xi_{g}\right)$ is the product of two even factors centered on $d_{p}=0$ and $d_{p}=-\xi_{g}$, respectively. As $\xi_{g}$ is changed, it is the shift of the profile peak from $d_{p}=0$ that is most evident in comparison to the variation in the width and asymmetry of the line profile. Further, since $\Im\left(d_{p} ; \xi_{g}\right)=\Im\left(-d_{p} ;-\xi_{g}\right)$ in Eq. (25) the shift of the profile peak will be in the opposite direction (but of the same magnitude) when switching from $\xi_{g}$ to $-\xi_{g}$. Different effects are observed for profiles with $q=0$. The example of $q=1.5$ is shown in Fig. 8(b). For $q=0, \mathfrak{I}\left(d_{p} ; \xi_{g}\right)$ is asymmetric, even for $\xi_{g}=0$, and also the magnitude of line shift depends on the sign of $\xi_{g}$. The plots in Fig. 9 illustrate all of the above-mentioned effects by showing the overall variation of the profile induced by changing the grating detuning, $\xi_{g}$ for different values of the probe detuning $d_{p}$.

The dependence of the line profile on the grating detuning for different values of $q$ shown in Figs. 8 and 9 suggests that quasibound states may be investigated in a different way by measuring the peak shift and the linewidth as a function of detunings. Since these parameters can be obtained from a fit of experimental data in a straightforward manner, the parametric TC-RFWM scheme represents an alternative and potentially efficient spectroscopic configuration that can take advantage of coherently controlling the signal line shape by modifying an experimental input parameter, in this case, the grating detuning $\xi_{g}$.

\section{CONCLUSIONS}

A theoretical prediction for the signal line profile observed by probing an isolated, quasibound state by means of TC-RFWM has been developed. In Sec. III, starting from first principles, the expression of the electric susceptibility that applies to this case has been derived. Even though the derivation has been specifically carried out for a resonant feature modeled as a configuration-interaction between a bound and continuum state leading to Eq. (2), the same procedure can be applied to any continuum resonance. Only two phenomenological inputs are needed: an appropriate model describing the probability of a transition from a lower state to a continuum resonance as a function of energy and a qualitative knowledge of the width of the distribution of continuum states involved. The first input consists of replacing $\left|\left\langle e\left|\mu_{\nu}\right| \eta\right)\right|^{2}$ with an appropriate dipole element that may be available, for example, from a MQDT treatment of a Rydberg series interacting with a continuum [26] or from the Wigner threshold law [32] for electron-ion photodetachment or photodissociation-photoassociation processes. The second input determines the selection of the resonant terms in the susceptibility. The final step in deriving a function for the line profile requires the calculation of an integral, which may be performed by numerical methods, if necessary. Parameters of physical interest can then be extracted from measured line profiles by a fitting procedure. Also, in principle, numerical deconvolution methods may be implemented to extract from the observed TC-RFWM spectra an experimental cross section for particular processes in which no model is a priori available.

In Secs. IV and V we have performed and discussed an analytic derivation of the TC-RFWM line profile in the case of a quasibound state described by a configurationinteraction. Two possible variations of the doubly resonant scheme were explored and the results demonstrate the ability to analyze observed line profiles in terms of the same physical parameters accessed by traditional linear techniques. Several distinctive aspects of the TC-RFWM profiles have been pointed out by comparing these results to the Fano profiles for quasibound resonances observed with linear spectroscopic techniques. Finally, in the parametric scheme, the 


$$
\begin{aligned}
& \mathbf{q}=\mathbf{0} \\
& \mathbf{r}=\mathbf{0}
\end{aligned}
$$

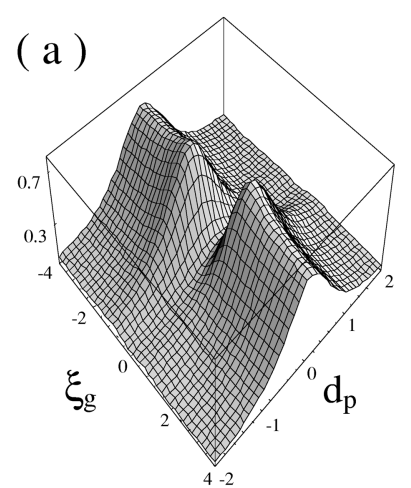

( b )

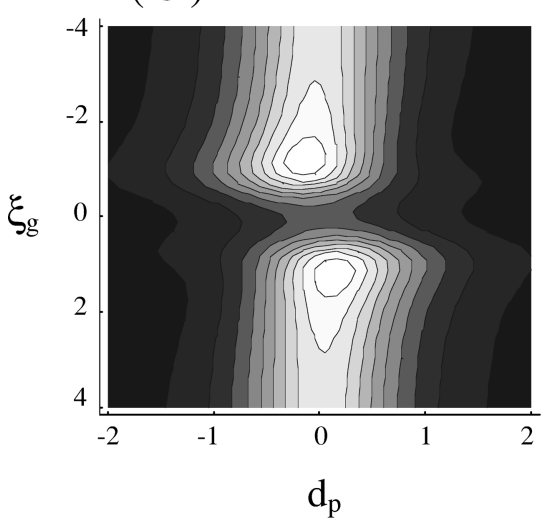

$q=1.5$

$\mathbf{r}=\mathbf{0}$

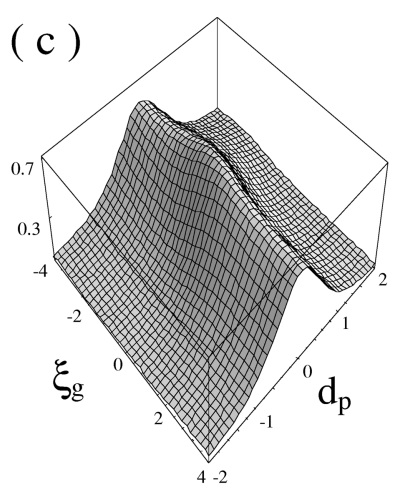

( d )

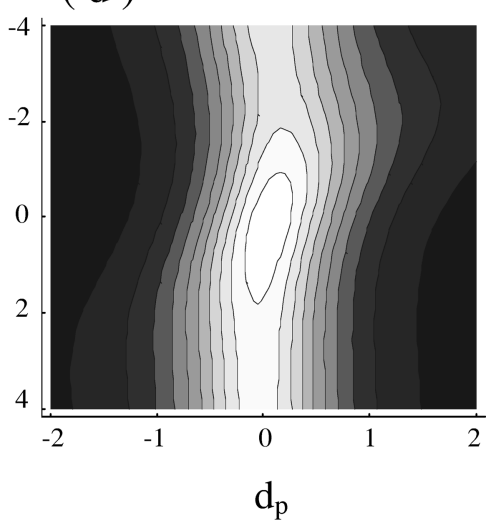

FIG. 9. TC-RFWM profiles for the parametric process depicted in Fig. 2 plotted as a function of the probe detuning $d_{p}$ and the grating detuning $\xi_{p}$. (a) and (c) 3D plots of $\Im\left(\xi_{g}, d_{p}\right)$ for $q=0$ and 1.5. (b) and (d) contour plot of the surfaces in (a) and (c), respectively.

possibility of controlling the line shape observed in scanning the probe frequency by using different grating detunings was highlighted as a possible approach for probing quasibound resonances.

\section{ACKNOWLEDGMENT}

This work was supported by the National Science Foundation, Grant No. PHY-9623569.

\section{APPENDIX A: DIRECTION OF THE PHASE-MATCHED WAVE VECTOR $\mathbf{k}_{s}$}

The direction of $\mathbf{k}_{s}$ in Eq. (14) is uniquely determined by the phase-matching condition $\omega_{s}=\omega_{p}$, introduced in Sec. III B and expressed in terms of wave vectors by Eq. (15). If the grating wave vector $\mathbf{g}=\mathbf{k}_{g_{1}}-\mathbf{k}_{g_{2}}$ is introduced [17], Eq. (15) may be rewritten as

$$
|\mathbf{g}|\left(|\mathbf{g}|+2 \frac{\omega_{p}}{c} \cos \beta\right)=0,
$$

where $\beta$ is the angle between $\mathbf{g}$ and $\mathbf{k}_{p}$, as shown in Fig. 4. Equation (A1) has two solutions: $\mathbf{g}=\mathbf{0}$ (i.e., $\mathbf{k}_{g_{1}}=\mathbf{k}_{g_{2}}$ ), implying $\mathbf{k}_{s}=\mathbf{k}_{p}$, and the solution

$$
|\mathbf{g}|+2 \frac{\omega_{p}}{c} \cos \beta=0
$$

If $\theta_{g}$ is the angle between $\mathbf{k}_{g_{1}}$ and $\mathbf{k}_{g_{2}}$, then

$$
|\mathbf{g}|=2 \frac{\omega_{g}}{c} \sin \frac{\theta_{g}}{2}
$$

and Eq. (A2) may be written as

$$
\omega_{g} \sin \frac{\theta_{g}}{2}+\omega_{p} \cos \beta=0
$$

Equation (A4) expresses the phase-matching condition in Eq. (15) as a geometrical constraint that relates the direction of incidence of the probe beam to the angle $\theta_{g}$. Finally, by calling $\theta_{p s}$ the angle between $\mathbf{k}_{p}$ and $\mathbf{k}_{s}$ and substituting Eq. (A3) into the vectorial identity

$$
\left|\mathbf{k}_{s}\right| \cos \theta_{p s}=\left|\mathbf{k}_{p}\right|+|\mathbf{g}| \cos \beta,
$$

Eq. (A4) may be cast in the form

$$
\frac{\omega_{g}}{\omega_{p}}=\frac{\sin \frac{\theta_{p s}}{2}}{\sin \frac{\theta_{g}}{2}}
$$




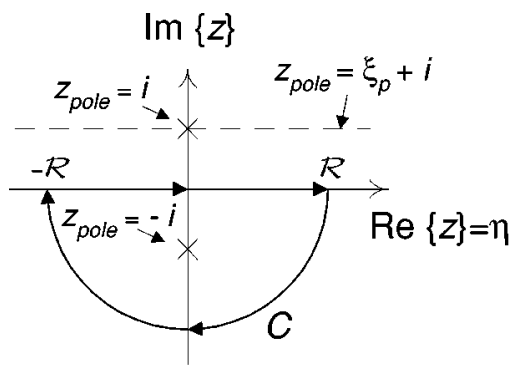

FIG. 10. Integration path in the complex plane used to evaluate Eq. (B1).

which is the well-known Bragg scattering condition [6] used to establish proper phase matching in laser-induced grating spectroscopy geometries.

\section{APPENDIX B: EVALUATION OF THE INTEGRAL IN EQ. (17)}

The evaluation of the integral in Eq. (17) may be performed by means of contour integration in the complex plane. The integration range $\mathcal{D}_{\eta}$ is taken over the entire interval $(-\infty, \infty)$. This choice, however, requires discussion. The extension of the integration interval to $(-\infty, \infty)$ causes a divergence in the integral in Eq. (17). The source of this unphysical behavior is the assumption that the parameters in the Fano profile in Eq. (2) are energy independent. This is an appropriate assumption near resonance for $\eta \sim 0$; however, far from resonance, the Fano profile actually goes to zero ensuring the expected convergence. This problem of convergence is addressed by calculating the Cauchy principal part of the integral in Eq. (17) for $\mathcal{D}_{\eta} \rightarrow(-\infty, \infty)$. This procedure is sufficient to make the integral converge, which confirms that the correction with respect to the exact situation may be thought of as small.

A complex variable $z$ is introduced such that $\eta=\operatorname{Re}(z)$, the integrand of Eq. (17) is evaluated in $z$, and three poles of first order are found: $z_{\text {pole }}= \pm i, \xi_{p}+i$. The semicircle path $C$ shown in Fig. 10 is chosen and the residue theorem is applied to give the identity

$$
\begin{aligned}
I & \equiv \mathrm{P} \int_{-\infty}^{\infty} d \eta \frac{1}{\xi_{p}-\eta+i}\left[p_{c} \frac{(q+\eta)^{2}}{1+\eta^{2}}+p_{u}\right] \\
& =-2 \pi i \operatorname{Res}(-i)+\lim _{\mathcal{R} \rightarrow \infty} \int_{-\pi}^{0} \frac{i e^{i \theta} d \theta}{-e^{i \theta}+\frac{\xi_{p}+i}{\mathcal{R}}} \\
& \times\left[p_{c} \frac{\left(\frac{q}{\mathcal{R}}+e^{i \theta}\right)^{2}}{\frac{1}{\mathcal{R}^{2}}+e^{i 2 \theta}}+p_{u}\right] .
\end{aligned}
$$

Here $\mathrm{P}$ indicates a principal-part integral, $\mathcal{R}$ is the radius of $C$, and $\operatorname{Res}(-i)$ is the residue of the integrand in $z=-i$. The final result is

$$
I=\frac{\left(q^{2}+2 r+1\right)-i\left[2 q+(1+r) \xi_{p}\right]}{\xi_{p}+2 i} .
$$

By calculating the absolute square of $I$ of Eq. (B2) the signal intensity in Eq. (18) is obtained. As long as $\mathcal{D}_{\eta}$ is a symmetric interval with respect to $\eta=0$, the transformation $\left\{q \rightarrow-q, \xi_{p} \rightarrow-\xi_{p}, \eta \rightarrow-\eta\right\}$ applied to Eq. (B1) results in $I$ becoming $-I^{*}$ and thus this transformation does not affect the calculation of $|I|^{2}$. This behavior explains the mirror symmetry for the profiles with opposite $q$ discussed in Sec. IV.

Finally, the integral in Eq. (21) may be evaluated in an identical manner by substituting $\left(\xi_{g}+d_{p}\right)$ for $\xi_{p}$ in Eq. (B1) and by using the same the integration procedure described above.
[1] T. Dreier and D. J. Rakestraw, Opt. Lett. 15, 72 (1990); R. L. Farrow, D. J. Rakestraw, and T. Dreier, J. Opt. Soc. Am. B 9, 1770 (1992); T. J. Buthenoff and E. A. Rohlfing, J. Chem. Phys. 97, 1595 (1992); E. F. McCormack, S. T. Pratt, P. M. Dehmer, and J. L. Dehmer, Chem. Phys. Lett. 211, 143 (1993).

[2] P. H. Vaccaro, in Molecular Dynamics and Spectroscopy by Stimulated Emission Pumping, edited by H. L. Dai and R. W. Field (World Scientific, Singapore, 1995), Vol. 4, p. 1.

[3] J. T. Fourkas and M. D. Fayer, Acc. Chem. Res. 25, 227 (1992)

[4] R. L. Farrow and D. J. Rakestraw, Science 257, 1894 (1992).

[5] G. Hall and J. Whitaker, J. Chem. Soc. Faraday Trans. 90, 1 (1994)

[6] P. N. Butcher and D. Cotter, The Elements of Nonlinear Optics (Cambridge University Press, Cambridge, 1990); R. W. Boyd, Nonlinear Optics (Academic, Boston, 1992).

[7] E. F. McCormack, S. T. Pratt, P. M. Dehmer, and J. L. Dehmer, Chem. Phys. Lett. 227, 656 (1994); J. Chem. Phys. 102, 4740 (1995); Aust. J. Phys. 49, 445 (1996).
[8] J. Ishii, K. Uehara, and K. Tsukiyama, J. Chem. Phys. 102, 9174 (1995).

[9] J. Geng, T. Kobayashi, and M. Takami, Chem. Phys. Lett. 266, 290 (1997).

[10] M. D. Wheeler, I. R. Lambert, and M. N. R. Ashfold, Chem. Phys. Lett. 229, 285 (1994).

[11] M. A. Buntine, D. W. Chandler, and C. C. Hayden, J. Chem. Phys. 102, 2718 (1995).

[12] T. Ebata, A. Okazaki, Y. Inokuchi, and N. Mikami, J. Mol. Struct. 352/353, 533 (1995).

[13] W. C. Hung, M. L. Huang, Y. C. Lee, and Y. P. Lee, J. Chem. Phys. 103, 9941 (1995).

[14] P. P. Radi, H. M. Frey, B. Mischler, A. P. Tzannis, P. Beaud, and T. Gerber, Chem. Phys. Lett. 265, 271 (1997).

[15] M. N. R. Ashfold, D. W. Chandler, C. C. Hayden, R. I. McKay, and A. J. R. Heck, Chem. Phys. 201, 237 (1995).

[16] B. Hemmerling, R. Bombach, and W. Hubschmid, Chem. Phys. Lett. 256, 71 (1996).

[17] T. J. Butenhoff and E. A. Rohlfing, J. Chem. Phys. 98, 5460 (1993); 98, 5469 (1993). 
[18] S. Williams, J. D. Tobiason, J. Dunlop, and E. A. Rohlfing, J. Chem. Phys. 102, 8342 (1995).

[19] T. Muller and P. H. Vaccaro, Chem. Phys. Lett. 266, 575 (1997).

[20] E. F. McCormack et al. (unpublished).

[21] S. Williams, E. A. Rohlfing, L. A. Rahn, and R. N. Zare, J. Chem. Phys. 106, 3090 (1997); and references therein.

[22] S. Williams, R. N. Zare, and L. A. Rahn, J. Chem. Phys. 101, 1072 (1994).

[23] R. T. Hodgson, P. P. Sorokin, and J. J. Wynne, Phys. Rev. Lett. 32, 343 (1974); J. A. Armstrong and J. J. Wynne, ibid. 33, 1183 (1974).

[24] M. Crance and L. Armstrong, J. Phys. B 15, 4637 (1982).

[25] G. S. Agarwal and P. Anantha Lakshmi, Phys. Rev. A 28, 3430 (1983).

[26] M. J. Seaton, Rep. Prog. Phys. 46, 97 (1983).
[27] U. Fano, Phys. Rev. 124, 1866 (1961).

[28] This model correctly applies to molecular, autoionizing Rydberg states for which the inequality $\gamma_{e} \ll \Gamma \ll \Delta E$ holds, where $\gamma_{e}$ is the decay rate of the excited state $|e\rangle$, from which the upward transition to a Rydberg level starts, and $\Delta E$ is the energy gap between two successive members of the Rydberg series.

[29] U. Fano and J. W. Cooper, Phys. Rev. 137, A1364 (1965).

[30] S. Mukamel, Principles of Nonlinear Optical Spectroscopy (Oxford University Press, New York, 1995), Chap. 6. For the general expression of $\chi^{(3)}$, see p. 151.

[31] Ab initio discussions of the diagrammatic perturbation theory may be found in J. G. Fujimoto and T. K. Yee, IEEE J. Quantum Electron. QE-22, 1215 (1986); Y. Prior, ibid. QE-20, 37 (1984), and references therein.

[32] E. P. Wigner, Phys. Rev. 73, 1002 (1948). 